\title{
On L- fuzzy generalized topology
}

\author{
Heba I. Mustafa \\ Mathematics Department, Faculty of Science, Zagazig University, Egypt
}

\begin{abstract}
In this paper, we introduce the concepts of L-fuzzy generalized neighborhood system(f-gns for short) and L-fuzzy generalized topology (fgt, for short)(where L is a fuzzy lattice) which are generalizations of generalized topology and neighborhood systems defined by Csaszar[5]. We also introduce and investigate with the help of these new concepts the concepts of L- $\left(\psi_{1}, \psi_{2}\right)$ continuity and L-fuzzy generalized continuity on f-gns. The relations between these concepts are investigated and several examples are presented. nifx
\end{abstract}

Keywords: Fuzzy lattice, L-fuzzy generalized topology, L-fuzzy generalized neighborhood systems, L-fuzzy generalized continuity

\section{1.. INTRODUCTION}

The usual notion of a set was generalized by Zadeh [21] when he introduced the notion of a fuzzy set which has useful and increasing applications in various fields.

Since many classes of information granules are lattice ordered $[2,14]$, lattice theory [10] has found renewed interesting applications in diverse areas such as mathematical morphology [12], fuzzy set theory [10,13,14], computational intelligence [17], automated decision making [15], and formal concept analysis [8]. Generalizing the concept of a fuzzy set, Goguen [10] in 1967 introduced the concept of L-fuzzy sets, where L is a fuzzy lattice. Recall that an L-fuzzy set is given by a mapping from a universe $\mathrm{X}$ to a set $\mathrm{L}$. In this setting, $\mathrm{L}$ has a mathematical structure that is at least a partially ordered set. Special emphasis was given to the case where L is a complete lattice. In 1973 Goguen[11] introduced the concept of L-fuzzy topological space as a generalization of a fuzzy topology introduced by Chang [3].

In $[4,5]$ Csaszar introduced the notion of generalized neighborhood systems and generalized topological spaces. He also introduced the notions of continuous functions and associated interior and closure operators on generalized neighborhood systems and generalized topological spaces. Moreover, he studied the simplest separation axioms for generalized topologies in $[6$, 7]. In [1, 16, 20,] G. Xun, J. Thomas and G. Abbaspour shall examine some topological properties such as, $\mu$-Compactness, the usual continuity, the net-continuity and the net-closure property for generalized topologies.

In this paper, we introduce the notion of generalized open Lfuzzy sets, called gamma open L-fuzzy subsets, by using monotonic mappings defined on the family of L-fuzzy subsets on a set X. We also, introduce the notions of L-fuzzy generalized neighborhood systems(fgns, for short) and L-fuzzy generalized topology (fgt, for short) which can be a generalization of neighborhood systems and generalized topology. L-fuzzy generalized system induces an L-fuzzy generalized neighborhood space. We introduce the new concepts of interior and closure on fgns and fgt on a set $\mathrm{X}$ and investigate some of their properties. Weaker forms of continuity are introduced by using these notions. We introduce the concept of L-fuzzy generalized continuity and L- $\left(\psi_{1}, \psi_{2}\right)$ continuity and we characterize some properties by the new interior and closure operators defined on fgns. We show that every
L- $\left(\psi_{1}, \psi_{2}\right)$ continuous function is L-fuzzy generalized continuous but the converse is not always true.

\section{2.. PRELIMINARIES}

Throughout this paper $\mathrm{X}$ and $\mathrm{Y}$ are non empty ordinary sets and $L=L\left(\leq, \vee, \wedge,,^{\prime}\right)$ denotes a fuzzy lattice, i.e, a complete completely distributive lattice with a smallest element 0 and a largest element $1(0 \neq 1)$ and with an order reversing involution $x \longrightarrow x^{\prime}(x \in L)$. We say that $x$ is the complement of a in $\mathrm{L}$. $\mathrm{L}$ is therefore continuous and spatial[9].

We denote by $L^{X}$ the lattice of $\mathrm{L}$-fuzzy subsets on $\mathrm{X}$.

Definition 2.1[9] An element $\mathrm{p}$ of $\mathrm{L}$ is called prime iff $p \neq 1$ and whenever $a, b \in L$ with $a \wedge b \leq p$ then $a \leq p$ or $b \leq p$. The set of all prime elements of $\mathrm{L}$ will be denoted by $\operatorname{pr}(\mathrm{L})$.

Warner[18]determined the prime elements of the fuzzy lattice $L^{X}$.We have $\operatorname{pr}\left(L^{X}\right)=\left\{x_{p}: x \in X\right.$ and $\left.p \in \operatorname{pr}(L)\right\}$ where, for each $x \in X$ and each $p \in \operatorname{pr}(L), x_{p}: X \longrightarrow L$ is the L-fuzzy subset defined by

$$
x_{p}(y)= \begin{cases}p & y=x \\ 0 & y \neq x\end{cases}
$$

These $x_{p}$ are called the L-fuzzy points of X.The set of all L-fuzzy points of $X$ will be denoted by $\operatorname{pt}\left(L^{X}\right)$.

Definition 2.2[19] For an L-fuzzy subset $\mu \in L^{X}$ and an L-fuzzy point $x_{p}$ of $\mathrm{X}$, we say that $x_{p}$ belongs to $\mu$, written $x_{p} \in \mu$ if $\mu(x) \not \leq p$.

Proposition 2.1[19] Let $\lambda, \mu \in L^{X}$ and $x_{p} \in p t\left(L^{X}\right)$. Then

(a) If $\lambda \leq \mu$,iff $\left(x_{p} \in \lambda \Longrightarrow x_{p} \in \mu\right)$.

(b) $x_{p} \in \lambda \wedge \mu$ iff $x_{p} \in \lambda$ and $x_{p} \in \mu$

(c) $x_{p} \in \bigvee_{i \in I} \lambda_{i}$ iff $x_{p} \in \lambda_{i}$ for some $i \in I$

Proposition 2.2[18] Every L-fuzzy subset $\mu \in L^{X}$ is the meet of L-fuzzy points of X.

Proposition 2.3[19] Let $f: X \longrightarrow Y, \lambda \in L^{X}$ and $\mu \in L^{X}$. Then the L-fuzzy subset $f(\lambda) \in L^{Y}$ is defined by $f(\lambda)(y)=\bigvee\left\{\lambda(x): x \in f^{-1}(y)\right\}$. and the L-fuzzy subset $f^{-1}(\mu) \in L^{X}$ is defined by $f^{-1}(\mu)(x)=(\mu \circ f)(x)$ for $x \in X$. The L-fuzzy set $f(\lambda)$ (resp. $f^{-1}(\mu)$ ) is called the image(resp., inverse image) of $\lambda$.

\section{3.. L-FUZZY GENERALIZED TOPOLOGY}

Definition 3.1 A mapping $\gamma: L^{X} \longrightarrow L^{Y}$ is a monotonic operator on X if it satisfies: For $\lambda, \mu \in L^{X}, \lambda \leq \mu \Longrightarrow \gamma(\lambda) \leq \gamma(\mu)$. In what follows $\Gamma(X)$ denotes the family of all monotonic operators on $\mathrm{X}$. 
Definition 3.2 Let $\gamma$ be a monotonic operator on a set $\mathrm{X}$. An L- fuzzy subset $\lambda \in L^{X}$ is said to be $\gamma$ open if $\lambda \leq \gamma(\lambda)$. One may notice that the empty L-fuzzy subset $\underline{0}$ is $\gamma$-open. Also the union of $\gamma$-open L-fuzzy subsets is $\gamma$-open L-fuzzy subset.

Definition 3.3 A family $\tau$ of L-fuzzy subset on $\mathrm{X}$ is said to be L-fuzzy generalized topology on $\mathrm{X}$ (fgt, for short) if $\underline{0} \in \tau$ and $\tau$ is closed under arbitrary union of L-fuzzy sets.

Proposition 3.1 Let $\gamma$ be a monotonic operator on a set $\mathrm{X}$.Then the family $\tau_{\gamma}$ of all $\gamma$-open L-fuzzy subsets is an fgt on $\mathrm{X}$. We say that $\tau_{\gamma}$ is the fgt on $\mathrm{X}$ induced by $\gamma$.

Proof: obvious.

In what follow any function $\gamma: L^{X} \longrightarrow L^{X}$ is assumed (otherwise stated) to be a monotonic operator on X. Also, by $\Im(X)$, we denote the collection of all fgt on a set X.

Proposition 3.2 Let $\tau \in \Im(X)$. Then there exists a monotonic operator $\gamma_{\tau}: L^{X} \longrightarrow L^{X}$ such that $\tau$ is the family of all $\gamma_{\tau}$-open L-fuzzy subsets. Furthermore, we have for $\lambda \in L^{X}$ (a) $\gamma_{\tau}(\underline{0})=0 \quad(b) \gamma_{\tau}(\lambda) \leq \lambda \quad(c) \gamma_{\tau}\left(\gamma_{\tau}(\lambda)\right)=\gamma_{\tau}(\lambda)$. We say that $\gamma_{\tau}$ is the monotonic operator on $\mathrm{X}$ induced by $\gamma$.

Proof: For $\lambda \in L^{X}$, define $\gamma_{\tau}(\lambda)=\bigvee\{\mu \in \tau: \mu \leq \lambda\}$. It is clear that $\gamma_{\tau}: L^{X} \longrightarrow L^{X}$ is a monotonic operator. Also $\gamma_{\tau}(\lambda) \in \tau$ and $\gamma_{\tau}(\lambda) \leq \lambda$.We show that $\tau$ is the family of all $\gamma_{\tau}$-open L-fuzzy subsets. Let $\lambda \in \tau$. Then $\gamma_{\tau}(\lambda)=\bigvee\{\mu \in \tau: \mu \leq \lambda\}=\lambda$. Hence $\lambda$ is $\gamma_{\tau}$-open. Let $\lambda$ be $\gamma_{\tau}$-open L-fuzzy set. Then $\lambda \leq \gamma_{\tau}(\lambda)=\bigvee\{\mu \in \tau: \mu \leq \lambda\} \leq \lambda$.Hence $\lambda=\gamma_{\tau}(\lambda)$ and $\lambda \in \tau$; since $\tau$ is a fgt on X.Thus $\tau$ is the family of all $\gamma_{\tau}$-open L-fuzzy subsets.

One may notice that for $\lambda \in L^{X}$, we have

$$
\lambda \in \tau \quad \text { iff } \quad \lambda=\gamma_{\tau}(\lambda)
$$

This proves (a) and (c) since $\underline{0}, \gamma_{\tau}(\lambda) \in \tau$.Also (b) follows from the definition of $\gamma_{\tau}$.

Proposition 3.3 Let $\tau \in \Im(X)$. Then $(a) \gamma_{\tau_{\gamma}}=\gamma$ and (b) $\tau_{\gamma \tau}=\tau$.

Proof: This is a direct consequence of Proposition3.1.

Let us consider another way for obtaining a fgt on X.

Definition 3.4 Let $\psi: p t\left(L^{X}\right) \longrightarrow P\left(L^{X}\right)$.Then $\psi$ is called a fuzzy generalized neighborhood operator and $\psi\left(x_{p}\right)$ (for $x \in X$ and $p \in \operatorname{pr}(L))$ is called a fuzzy generalized neighborhood system(fgns for short) for $x_{p}$ if it satisfies

$$
\mu \in \psi\left(x_{p}\right) \Longrightarrow x_{p} \in \mu \text {. }
$$

In this case,we say that $\mu \in \psi\left(x_{p}\right)$ is a fuzzy generalized neighborhood of $x_{p}$ (fgn for short). By $\Psi(X)$ we denote the collection of all fuzzy generalized neighborhood operators on $\mathrm{X}$.

Proposition 3.4 Let $\psi \in \Psi(X)$. Then there exists a fgt $\tau_{\psi}$, on $\mathrm{X}$, such that

$$
\mu \in \tau_{\psi} \text { iff } \forall x_{p} \in \mu \exists \lambda \in \psi\left(x_{P}\right) \text { such that } \lambda \leq \mu
$$

Proof: It is clear that $\underline{0} \in \tau_{\psi}$. Let $\mu_{i} \in \tau_{\psi}$ and let $x_{p} \in \bigvee \mu_{i}$. Then $x_{p} \in \mu_{i}$ for some i. Hence $\exists \lambda_{i} \in \psi\left(x_{p}\right)$ s.t $\lambda_{i} \leq$ $\mu_{i}$.Thus $\exists \lambda_{i} \in \psi\left(x_{p}\right)$ s.t $\lambda_{i} \leq \bigvee \mu_{i}$. In otherwords $\bigvee \mu_{i} \in \tau_{\psi}$. Consequently, $\tau_{\psi}$ is a fgt on $\mathrm{X}$.
Proposition 3.5 Let $\tau \in \Im(X)$. Then there exists an Lfuzzy generalized neighborhood operator $\psi_{\tau}$, on $\mathrm{X}$, such that

$$
\mu \in \tau \text { iff } \forall x_{p} \in \mu \exists \lambda \in \psi_{\tau}\left(x_{p}\right) \text { such that } \lambda \leq \mu
$$

We say that $\psi_{\tau}$ is the L-fuzzy generalized operator induced by $\tau$.

Proof: Define $\lambda \in \psi_{\tau}\left(x_{p}\right) \Longleftrightarrow x_{p} \in \lambda \in \tau$. It is clear that $\psi_{\tau}$ is a fgn operator on X. Now, let $\mu \in \tau$ and let $x_{p} \in \mu$. Then $\exists \mu \in \psi_{\tau}\left(x_{p}\right)$ such that $\mu \leq \mu$.

Proposition 3.6 Let $\tau \in \Im(X)$. Then $\tau_{\psi_{\tau}}=\tau$.

Proof: This is a direct consequence of Propositions 3.4 and 3.5 .

Definition 3.5 Let $\tau \in \Im(X)$ and $\lambda \in L^{X}$. The $\tau$-interior and $\tau$-closure of $\lambda$ are defined and denoted respectively,by

$i_{\tau}(\lambda)=\vee\{\mu \in \tau: \mu \leq \lambda\}$ and $c_{\tau}(\lambda)=\wedge\{\grave{\mu} \in \tau: \mu \geq \lambda\}$ If $\tau=\tau_{\gamma}$, we write $i_{\gamma}$ for $i_{\tau_{\gamma}}$ and $c_{\gamma}$ for $c_{\tau_{\gamma}}$. For a fuzzy generalized neighborhood operator $\psi$, we write $i_{\psi}$ (resp. $c_{\psi}$ ) instead of $i_{\tau_{\psi}}$ (resp. $c_{\tau_{\psi}}$ ).

Definition 3.6 Let $\psi \in \Psi(X)$ and $\lambda \in L^{X}$.The two operators $I_{\psi}, I_{\psi}: L^{X} \longrightarrow L^{X}$ are defined by

$$
\begin{gathered}
(i) I_{\psi}(\lambda)=\bigvee\left\{x_{p}^{\prime} \in L^{X}: \exists \mu \in \psi\left(x_{p}\right) \text { s.t } \mu \leq \lambda\right\} \\
\text { (ii) } \gamma_{\psi}(\lambda)=\bigwedge\left\{x_{p} \in L^{X}: \exists \mu \in \psi\left(x_{p}\right) \text { s.t } \mu \leq \lambda^{\prime}\right\}
\end{gathered}
$$

Proposition 3.7 Let $\psi \in \Psi(X)$ and $\lambda \in L^{X}$. Then

(a) $I_{\psi}$ and $\gamma_{\psi} \in \Gamma(X)$,i.e. $I_{\psi}$ and $\gamma_{\psi}$ are monotonic operators.

(b) $I_{\psi}(\lambda) \leq \lambda$ and $\gamma_{\psi}(\lambda) \geq \lambda$

(c) $\gamma_{\psi}(\lambda)=\left(I_{\psi}\left(\lambda^{\prime}\right)\right)^{\prime}$

(d) $i_{\psi}(\lambda) \leq I_{\psi}(\lambda)$ and $c_{\psi}(\lambda) \geq \gamma_{\psi}(\lambda)$

Proof:(a) Obvious. (b) Let $y_{p_{1}} \in I_{\psi}(\lambda)$, then there exists $x_{p} \in L^{X}$ and $\mu \in \psi\left(x_{p}\right)$ such that $y_{p_{1}} \in x_{p}^{\prime}$, where $x_{p}^{\prime}(y)=\left\{\begin{array}{ll}p^{\prime} & \text { if } y=x \\ 0 & \text { if } y \neq x\end{array}\right.$ and $\mu \leq \lambda$. Hence $x^{\prime}{ }_{p}(y) \not \leq p_{1}$, i.e $x_{p}^{\prime}(y)=p^{\prime} \not \leq p_{1}$. Thus $y=x$ and $y_{p_{1}}=x_{p_{1}} \in x_{p}^{\prime}$. Since $x_{p} \in \mu$, then $\mu(x) \leq \leq p$ and hence $\mu^{\prime}(x) \leq p$. In fact, assume that $\mu^{\prime}(x) \leq p$. Since $\mu(x) \wedge \mu^{\prime}(x)=0 \leq p$ and $p \in \operatorname{pr}(L)$, then $\mu(x) \leq p$ or $\mu^{\prime}(x) \leq p$, a contradiction. Therefore $\mu^{\prime}(x) \leq p \nsupseteq p_{1}{ }^{\prime}$ and hence $\mu(x) \not \leq p_{1}$. So $x_{p_{1}} \in \mu \leq \lambda$ and $x_{p_{1}} \in \bar{\lambda}$. Consequently, $I_{\psi}(\lambda) \leq \lambda$. Similarly, we can show that $\gamma_{\psi}(\lambda) \geq \lambda$

(c) $\left(I_{\psi}\left(\lambda^{\prime}\right)\right)^{\prime}=\left(\bigvee\left\{x_{p}^{\prime} \in L^{X}: \exists \mu \in \psi\left(x_{p}\right) \text { s.t } \mu \leq \lambda^{\prime}\right\}^{\prime}\right.$ $=\bigwedge\left\{x_{p} \in L^{X}: \exists \mu \in \psi\left(x_{p}\right)\right.$ s.t $\left.\mu \leq \lambda^{\prime}\right\}=\gamma_{\psi}(\lambda)$.

(d)Let $y_{p_{1}} \in i_{\psi}(\lambda)=\bigvee\left\{\mu \in \tau_{\psi}: \mu \leq \lambda\right\}$. Hence $\exists$ $\mu \in \tau_{\psi}$ s.t $y_{p_{1}} \in \mu \leq \lambda$. So, $\exists \delta \in \psi\left(x_{p}\right)$ s.t $y_{p_{1}} \in \delta \leq \mu \leq \lambda$. Since $y_{p_{1}}^{\prime}(y)=p_{1}^{\prime} \not \leq p_{1}$, then $\exists y_{p_{1}}^{\prime} \in L^{X}$ s.t $y_{p_{1}} \in y_{p_{1}}^{\prime}$ and $y_{p_{1}} \in \delta \leq \mu$. Consequently, $y_{p_{1}} \in I_{\psi}(\lambda)$. Hence $i_{\psi}(\lambda) \leq I_{\psi}(\lambda)$. The other part follows directly from (c).

Remark 3.1 In general, $I_{\psi}(\lambda) \neq i_{\psi}(\lambda)$ and $\gamma_{\psi}(\lambda) \neq c_{\psi}(\lambda)$. The following example illustrates this fact.

Example 3.1 Let $X=\{x, y\}$ and $L=\{0, a, b, c, d, e, f, 1\}$ be a fuzzy lattice described as Figure 1 


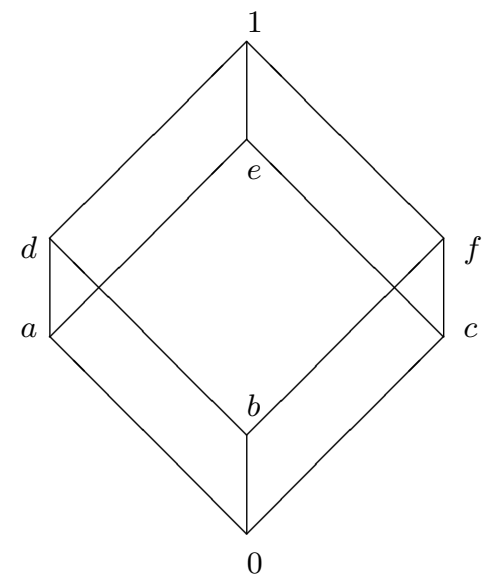

figure 1

It is clear that $\operatorname{pr}(L)=\{d, e, f\}$. So $\operatorname{pt}\left(L^{X}\right)=$ $\left\{x_{d}, x_{e}, x_{f}, y_{d}, y_{e}, y_{f}\right\}$. Let us define $\psi: p t\left(L^{X}\right) \longrightarrow P\left(L^{X}\right)$ by $\psi\left(x_{d}\right)=\left\{\mu_{6}, \mu_{7}\right\}, \psi\left(x_{e}\right)=\left\{\mu_{7}\right\}, \psi\left(x_{f}\right)=\left\{\mu_{8}, \mu_{5}, \mu_{6}\right\}$ and $\psi\left(y_{d}\right)=\psi\left(y_{e}\right)=\psi\left(y_{f}\right)=0$. Where $\mu_{1}(x)=0$, $\mu_{2}(x)=a, \mu_{3}(x)=b, \mu_{4}(x)=c, \mu_{5}(x)=d, \mu_{6}(x)=e$, $\mu_{7}(x)=f, \mu_{8}(x)=1$ and $\mu_{i}(y)=0, \mathrm{i}=1,2, \ldots \ldots, 8$.

Then $\tau_{\psi}=\left\{\mu_{1}, \mu_{6}, \mu_{7}, \mu_{8}\right\}$. It is clear that $\mu_{1} \in \tau_{\psi}$. Also $\mu_{2} \notin \tau_{\psi}$. In fact, $\mu_{2}(x)=a \not \leq f$ and hence $x_{f} \in \mu_{2}$. So, $\nexists$ $\lambda \in \psi\left(x_{f}\right) \leq x_{f}$. Similarly, $\mu_{3}, \mu_{4}, \mu_{5} \notin \tau_{\psi}$. Now, $\mu_{6} \in \tau_{\psi}$. In fact, $x_{d}, x_{f} \in \mu_{6}$ and $\exists \lambda=\mu_{6} \in \psi\left(x_{d}\right)$ and $\psi\left(x_{f}\right)$ s.t $\lambda \leq \mu_{6}$. Similarly, $\mu_{7}, \mu_{8} \in \tau_{\psi}$.

Now, we show that $i_{\psi}\left(\mu_{5}\right) \neq I_{\psi}\left(\mu_{5}\right)$. In fact, $i_{\psi}\left(\mu_{5}\right)=\bigvee\left\{\lambda \in \tau_{\psi}: \lambda \leq \mu_{5}\right\}=\mu_{1}$. Also $I_{\psi}\left(\mu_{5}\right)=\bigvee\left\{x_{p}^{\prime} \in L^{X}: \exists \lambda \in \psi\left(x_{p}\right)\right.$ s.t $\left.\lambda \leq \mu_{5}\right\}=\mu_{2}$, since $\exists \lambda=\mu_{5} \in \psi\left(x_{f}\right)$ s.t $\lambda \leq \mu_{5}$. Hence $x_{f}^{\prime} \in I_{\psi}\left(\mu_{5}\right)$ and thus $\mu_{2}=x_{f}^{\prime}=I_{\psi}\left(\mu_{5}\right)$. Consequently, $I_{\psi}\left(\mu_{5}\right)=\mu_{2} \neq \mu_{1}=i_{\psi}\left(\mu_{5}\right)$.

We show that $\gamma_{\psi}\left(\mu_{4}\right) \neq c_{\psi}\left(\mu_{4}\right)$. Since $\tau_{\psi}^{\prime}=\left\{\mu_{1}, \mu_{2}, \mu_{3}, \mu_{8}\right\}$, then $c_{\psi}\left(\mu_{4}\right)=\bigwedge\left\{\lambda \in \tau_{\psi}^{\prime}: \mu_{4} \leq \lambda^{\prime}\right\}=\mu_{8}$. Also, $\gamma_{\psi}\left(\mu_{4}\right)=$ ${ }^{\prime}\left(I_{\psi}\left(\mu_{4}^{\prime}\right)^{\prime}\right)=\left(I_{\psi}\left(\mu_{5}\right)\right)^{\prime}=\mu_{2}^{\prime}=\mu_{7} \neq \mu_{8}=c_{\psi}\left(\mu_{4}\right)$.

Proposition 3.8 Let $\tau \in \Im(X)$. Then $I_{\psi_{\tau}}=i_{\psi_{\tau}}$ and $\gamma_{\psi_{\tau}}=c_{\psi_{\tau}}$

Proof: Let $y_{p_{1}} \in I_{\psi_{\tau}}(\lambda)$. Then $\exists x_{p} \in L^{X}$ and $\mu \in \psi_{\tau}\left(x_{p}\right)$ s.t $y_{p_{1}} \in x_{p}^{\prime}$ and $\mu \leq \lambda$. Hence $y=x$ and $x_{p}^{\prime}(x)=p^{\prime} \not \leq p_{1}$. Therefore, $\mu \in \tau_{\psi_{\tau}}=\tau$. Since $x_{p} \in \mu$, then $\mu(x) \not \leq p$ and hence $\mu^{\prime}(x) \leq p \nsupseteq p_{1}^{\prime}$. So $\mu(x)$ $\leq p_{1}$ and therefore $x_{p_{1}} \in \mu \leq \lambda$. Consequently, $x_{p_{1}} \in i_{\psi_{\tau}}(\lambda)$.The inclusion $i_{\psi_{\tau}} \subset I_{\psi_{\tau}}$ follows from Proposition 3.6. The other equality results by considering the complements.

\section{4.. L-FUZZY GENERALIZED CONTINUITY}

Definition 4.1 Let $\tau_{1} \in \Im\left(X_{1}\right)$ and $\tau_{2} \in \Im\left(X_{2}\right)$. Let $f$ : $X_{1} \longrightarrow X_{2}$. Then $\mathrm{f}$ is called $\left(\tau_{1}, \tau_{2}\right)$ continuous if

$$
\mu \in \tau_{2} \Longrightarrow f^{-1}(\mu) \in \tau_{1}
$$

We obtain another (more general) kind of L-fuzzy generalized continuity.

Definition 4.2 Let $X_{1}$ and $X_{2}$ be non empty sets and $f: X_{1} \longrightarrow X_{2}$. Let $\psi_{1} \in \Psi\left(X_{1}\right)$ and $\psi_{2} \in \Psi\left(X_{2}\right)$. Then $\mathrm{f}$ is called $\left(\psi_{1}, \psi_{2}\right)$ continuous if

$\forall x_{p} \in p t\left(L^{X}\right)$ and $\mu \in \psi_{2}(f(x))_{p} \exists \lambda \in \psi_{1}\left(x_{p}\right)$ s.t $f(\lambda) \leq \mu$
The following propositions give the relation between the above two kinds of L-fuzzy generalized continuity.

Proposition 4.1 Let $X_{1}$ and $X_{2}$ be non empty sets and $f: X_{1} \longrightarrow X_{2}$. Let $\psi_{1} \in \Psi\left(X_{1}\right)$ and $\psi_{2} \in \Psi\left(X_{2}\right)$. If $\mathrm{f}$ is $\left(\psi_{1}, \psi_{2}\right)$ continuous, then $\mathrm{f}$ is $\left(\tau_{\psi_{1}}, \tau_{\psi_{2}}\right)$ continuous.

Proof: Let $\mu \in \tau_{\psi_{2}}$ and $x_{p} \in f^{-1}(\mu)$. Then $\mu(f(x))=\left(f^{-1}(\mu)\right)(x) \quad \not \quad p$. Hence, $(f(x))_{p} \in \mu$. Since $\mu \in \tau_{\psi_{2}}$, then $\exists \lambda \in \psi_{2}(f(x))_{p}$ s.t $\lambda \leq \mu$. Since $\mathrm{f}$ is $\left(\psi_{1}, \psi_{2}\right)$ continuous, then $\exists \delta \in \psi_{1}\left(x_{p}\right)$ s.t $f(\delta) \leq \lambda \leq \mu$. Hence $\delta \leq f^{-1}(\mu)$ and consequently $f^{-1}(\mu) \in \tau_{\psi_{1}}$.

Remark 3.1The converse of the previous proposition is not generally true. The following example illustrates this idea.

Example 4.1 Let $X=\{x, y\}$ and $L=\{0, a, b, c, d, e, f, 1\}$ be a fuzzy lattice described as Figure 1. Let $\psi_{1}, \psi_{2} \in \Psi(X)$ be defined as follows

$\psi_{1}\left(x_{d}\right)=\psi_{2}\left(x_{d}\right)=\left\{\mu_{6}, \mu_{7}\right\}, \psi_{1}\left(x_{e}\right)=\psi_{2}\left(x_{e}\right)=\left\{\mu_{7}\right\}$, $\psi_{1}\left(x_{f}\right)=\left\{\mu_{8}, \mu_{6}\right\}, \psi_{2}\left(x_{f}\right)=\left\{\mu_{8}, \mu_{5}, \mu_{6}\right\}$ and $\psi_{i}\left(y_{d}\right)=\psi_{i}\left(y_{e}\right)=\psi_{i}\left(y_{f}\right)=0(\mathrm{i}=1,2)$.

It is clear that $\tau_{\psi_{1}}=\tau_{\psi_{2}}=\left\{\mu_{1}, \mu_{8}, \mu_{6}, \mu_{7}\right\}$. Let $f=i d_{X}$ i.e, $f: X \longrightarrow X$ such that $f(x)=x$ for all $x \in X$. Hence $f(\mu)=\mu$ for all $\mu \in L^{X}$ and so $f\left(x_{f}\right)=(f(x))_{f}=x_{f}$. It is clear that $\mathrm{f}$ is $\left(\tau_{\psi_{1}}, \tau_{\psi_{2}}\right)$ continuous. Now, $\mu_{5} \in \psi_{2}\left(f\left(x_{f}\right)\right)=\psi_{2}\left(x_{f}\right)$, but $\nexists \lambda \in \psi_{1}\left(x_{f}\right)$ s.t $f(\lambda)=\lambda \leq \mu_{5}$. Consequently, $\mathrm{f}$ is not $\left(\psi_{1}, \psi_{2}\right)$ continuous.

Proposition 4.2 Let $X_{1}$ and $X_{2}$ be non empty sets and $f: X_{1} \longrightarrow X_{2}$. Let $\psi_{1} \in \Psi\left(X_{1}\right)$ and $\psi_{2} \in \Psi\left(X_{2}\right)$. If $\mathrm{f}$ is $\left(\tau_{\psi_{1}}, \tau_{\psi_{2}}\right)$ continuous and $\psi_{2}=\psi_{\tau_{1}}$ for some fgt $\tau_{1}$ on $X_{2}$. then $\mathrm{f}$ is $\left(\psi_{1}, \psi_{2}\right)$ continuous.

Proof:Let $\mu \in \psi_{2}\left(f(x)_{p}\right)=\psi_{\tau_{1}}\left(f(x)_{p}\right.$. Hence $f\left(x_{p}\right) \in \mu$ and $\mu \in \tau_{1}$. By Proposition 3.6, $\tau_{\psi_{2}}=\tau_{\psi_{\tau_{1}}}=\tau_{1}$ and thus $\mu \in \tau_{\psi_{2}}$. So, $x_{p} \in f^{-1}(\mu) \in \tau_{\psi_{1}}$ because $\mathrm{f}$ is $\left(\tau_{\psi_{1}}, \tau_{\psi_{2}}\right)$ continuous. Therefore $\exists \lambda \in \psi_{1}\left(x_{p}\right)$ s.t $\lambda \leq f^{-1}(\mu)$. Then $f(\lambda) \leq \mu$ and consequently, $\mathrm{f}$ is $\left(\psi_{1}, \psi_{2}\right)$ continuous.

Proposition 4.3 Let $X_{1}$ and $X_{2}$ be non empty sets and $f: X_{1} \longrightarrow X_{2}$. Let $\psi_{1} \in \Psi\left(X_{1}\right)$ and $\psi_{2} \in \Psi\left(X_{2}\right)$. The following statements are equivalent

(a) $\mathrm{f}$ is $\left(\psi_{1}, \psi_{2}\right)$ continuous

(b) $f\left(\gamma_{\psi_{1}}(\mu)\right) \leq \gamma_{\psi_{2}}(f(\mu)) \forall \mu \in L^{X_{1}}$

(c) $\gamma_{\psi_{1}}\left(f^{-1}(\lambda)\right) \leq f^{-1}\left(\gamma_{\psi_{2}}(\lambda)\right) \forall \lambda \in L^{X_{2}}$

Proof: $(a) \Longrightarrow(b)$ Let $x_{p} \in \gamma_{\psi_{1}}(\mu)$ and assume that $f\left(x_{p}\right) \notin \gamma_{\psi_{2}}(f(\mu))=\left(I_{\psi_{2}}(f(\dot{\mu}))\right)^{\prime}$. Hence $f\left(x_{p}\right) \in I_{\psi_{2}}\left(f\left(\mu^{\prime}\right)\right)$ and so $\exists \lambda \in \psi_{2}\left(f\left(x_{p}\right)\right)$ s.t $\lambda \leq$ $f\left(\mu^{\prime}\right)=(f(\mu))^{\prime}$. Therefore $\lambda \wedge f(\mu)=0$. Since $\mathrm{f}$ is $\left(\psi_{1}, \psi_{2}\right)$ continuous, then $\exists \delta \in \psi_{1}\left(x_{p}\right)$ s.t $f(\delta) \leq \mu$. Hence $f(\delta) \wedge f(\mu) \leq \lambda \wedge f(\mu)=0$ and therefore $f(\delta \wedge \mu)=0$. Thus $\delta \wedge \mu=0$ i.e, $\delta \leq \mu$. Then $\exists x_{p}^{\prime} \in L^{X}$ and $\delta \in \psi_{1}\left(x_{p}\right)$ s.t $x_{p} \in x_{p}^{\prime}$ and $\delta \leq \mu^{\prime}$. So $x_{p} \in I_{\psi_{1}}\left(\mu^{\prime}\right)$ and thus $x_{p} \notin\left(I_{\psi_{1}}\left(\mu^{\prime}\right)\right)^{\prime}=\gamma_{\psi_{1}}(\mu)$. Consequently, $f\left(x_{p}\right) \notin f\left(\gamma_{\psi_{1}}(\mu)\right)$.

(b) $\Longrightarrow(c)$ Let $\mu=f^{-1}(\lambda)$. Then by (b) $f\left(\gamma_{\psi_{1}}(\mu)\right) \leq$ $\gamma_{\psi_{2}}(f(\mu))=\gamma_{\psi_{2}}\left(f\left(f^{-1}(\lambda)\right)\right) \leq \gamma_{\psi_{2}}(\lambda)$. Hence $\gamma_{\psi_{1}}(\mu)=\gamma_{\psi_{1}}\left(f^{-1}(\lambda)\right) \leq f^{-1}\left(\gamma_{\psi_{2}}(\lambda)\right)$.

$(c) \Longrightarrow(a)$ Let $\lambda \in \psi_{2}\left(f\left(x_{p}\right)\right.$, then $f\left(x_{p}\right) \in \lambda$. Let $\mu=\lambda^{\prime}$, then $f\left(x_{p}\right) \notin \gamma_{\psi_{2}}(\mu)$. In fact, $\exists \lambda \in$ $\psi_{2}\left(f\left(x_{p}\right)\right)$ s.t $\lambda=\mu^{\prime} \leq \mu^{\prime}$ but $f\left(x_{p}\right) \notin f\left(x_{p}\right)$. So, $x_{p} \notin f^{-1}\left(\gamma_{\psi_{2}}(\mu)\right)$. By (c), $x_{p} \notin \gamma_{\psi_{1}}\left(f^{-1}(\mu)\right)$ and hence, $\exists \delta \in \psi_{1}\left(x_{P}\right)$ s.t $\delta \leq\left(f^{-1}(\mu)\right)^{\prime}$. Thus $\delta \wedge f^{-1}(\mu)=0$ and 
then $f(\delta) \wedge \mu=0$. Hence $f(\delta) \leq \mu^{\prime}=\lambda$ and consequently, $\mathrm{f}$ is $\left(\psi_{1}, \psi_{2}\right)$ continuous.

\section{5.. REFERENCES}

[1] G. Abbaspour and A. Taghavi, A note on generalized topology, international mathematical forum, 6(1)(2011), 19-24.

[2] A. Bargiela, W. Pedrycz, Granular Computing: An Introduction. Kluwer Academic Publishers, Hingham, MA (2003).

[3] C. L. Chang, Fuzzy topological spaces, J Math. Anal. Appl., 24(1968), 182-190.

[4] A. Csaszar, Generalized open sets, Acta Math. Hungar., 75(1997), 65-87.

[5] A. Csaszar, Generalized continuity, Acta Math. Hungar., 96(2002), 351-357.

[6] A. Csaszar, Separation axioms for generalized topologies, Acta Math Hungar, 104(2004), 63-69.

[7] A. Csaszar, Extremally disconnected generalized topologies, Annales Univ Budapest, Sectio Math, 47(2004), 151-161.

[8] B. Ganter, P. Wille, formal concept analysis, springer, Berlin, Germany, 1999.

[9] G. Gerz et. al, A compendium of continuous lattices, (Springer, Berlin, 1980).

[10] J. A. Goguen, L-fuzzy subsets, J Math. Anall. Appl. ,18(1967), 115-174.

[11] J. A. Goguen, The fuzzy Tychonof theorem, J Math.Anall. Appl., 43(1973), 734-742.
[12] H. Heijmans, Morphological Image Operators. Academic Press, New York, NY (1994).

[13] J. Jarvinen, Set operations for L-fuzzy sets. In: Rough Sets amd Intelligent Sys- tem radigms. Volume 4585 of Lecture Notes in Computer Science. Springer Berlin / Heidelberg (2007), 221-229.

[14] J. Jarvinen, Lattice theory for rough sets. In: Transactions on Rough Sets VI. Volume 4374 of Lecture Notes in Computer Science. Springer Berlin / Heidelberg, (2007), 400498.

[15] Y. Raun, J. Qin, Lattice-valued logic, springer, Heidelberg, Germany,2003.

[16] J. Thomas, S. Jacob, $\mu$-Compactness in Generalized Topological Spaces,Journal of Advanced Studies in Topology, 3(3)(2012), 18-22.

[17] G. Urcid, N. Valdiviezo, J. Ritter, Lattice algebra approach to color image segmentation. Journal of Mathematical Imaging and Vision 42(2-3) (2012), 150-162.

[18] M. W. Warner, frame fuzzy points and membership, fuzzy set and systems 42(1991)103-110.

[19] M. W. Warner, fuzzy topology with respect to continuous lattices, fuzzy set and systems, 35(1990), 85-91.

[20] G. Xun and G. Ying, $\mu$ separations in generalized topological spaces, Appl. Math. J. Chinese Univ, 25(2010), 243-252.

[21] L. A. Zadeh, fuzzy sets, Information and control, (1995), 353-383. 